\title{
Dysregulation of microRNA-125b is involved in the pathogenesis of post-operative infection via modulating the STAT3/procalcitonin (PCT) signaling pathway
}

\section{Type}

Research paper

\author{
Keywords \\ procalcitonin, PCT, miRNA-125b, post-operative infection
}

\begin{abstract}
Introduction

Procalcitonin (PCT) has been reported to function as a predictive biomarker of post-operative infection. In this study, we aimed to explore the mechanisms underlying the regulation of PCT expression.
\end{abstract}

\begin{abstract}
Material and methods
72 children diagnosed with post-operative infection and 58 children without post-operative infection were recruited in this study. Computational analysis, luciferase assay, real-time PCR, Western-blot analysis, and assays of post-operative C-reactive protein (CRP), erythrocyte sedimentation rate (ESR) and PCT were performed to investigate the mechanisms underlying the regulation of PCT expression.
\end{abstract}

\section{Results}

MiR-125b was found to repress STAT3 expression with putative binding sites in 3'UTR of STAT3. The levels of PCT and miR-125b in non-infection group remained stable from day 0 to day 5 , while the level of PCT was increased in the infection group along with a decreased level of miR-125b from day 1 to day 5 . The post-operative levels of CRP and ESR in both non-infection and infection groups were evidently increased in a time-dependent manner, but the levels of miR-106b and miR-20a in both non-infection and infection groups remained stable. The area under the curve (AUC) values of PCT, CRP, ESR, miR-125b, miR-106b and miR-20a demonstrated that only miR-125b and PCT were involved in infection. Transfection with miR-125b reduced STAT3 expression, while the activation of STAT3 by lipopolysaccharide (LPS) treatment up-regulated PCT production. Finally, miR-125b down-regulated the expression of PCT by targeting STAT3.

\section{Conclusions}

Taken together, we suggested that miR-125b was involved in the prognosis and diagnosis of posteroperative infection by modulating the signaling pathway of miR-125b/STAT3/PCT. 


\section{Dysregulation of microRNA-125b is involved in the pathogenesis of post-operative infection via modulating the STAT3/procalcitonin (PCT) signaling pathway}

Li Wang ${ }^{1}$, Mingzhu Yang ${ }^{2}$, Xuan Jin ${ }^{3 *}$

1. Laboratory, Affiliated Hospital of Shaanxi University of Chinese Medicine, Xianyang, Shaanxi, 712000, China

2. Pediatrics Department, Xianyang Central Hospital, Xianyang, Shaanxi, 712000 , China

3. Pediatrics Department, Affiliated Hospital of Shaanxi University of Chinese Medicine, Xianyang, Shaanxi, 712000, China

*Corresponding author: Xuan Jin

Institution: Pediatrics Department, Affiliated Hospital of Shaanxi University of Chinese Medicine, Xianyang, Shaanxi, 712000, China

Address: No.2, Yangxi Road, Xianyang, Shaanxi, 712000, China

Email: docknifes@yeah.net

\section{Abstract}

Background: Procalcitonin (PCT) has been reported to function as a predictive biomarker of post-operative infection. In this study, we aimed to explore the mechanisms underlying the regulation of PCT expression. Method: 72 children diagnosed with post-operative infection and 58 children without post-operative infection were recruited in this study. Computational analysis, luciferase assay, real-time PCR, Western-blot analysis, and assays of post-operative C-reactive protein (CRP), erythrocyte sedimentation rate (ESR) and PCT were performed to investigate the mechanisms underlying the regulation of PCT expression. Result: MiR-125b was found to repress STAT3 expression with putative binding sites in 3'UTR of STAT3. The levels of PCT and miR-125b in non-infection group remained stable from day 0 to day 5, while the level of PCT was increased in the infection group along with a decreased level of miR-125b from day 1 to day 5 . The post-operative levels of CRP and ESR in both non-infection and infection groups were evidently increased 
in a time-dependent manner, but the levels of miR-106b and miR-20a in both noninfection and infection groups remained stable. The area under the curve (AUC) values of PCT, CRP, ESR, miR-125b, miR-106b and miR-20a demonstrated that only miR-125b and PCT were involved in infection. Transfection with miR-125b reduced STAT3 expression, while the activation of STAT3 by lipopolysaccharide (LPS) treatment up-regulated PCT production. Finally, miR-125b down-regulated the expression of PCT by targeting STAT3.

Conclusion: Taken together, we suggested that miR-125b was involved in the prognosis and diagnosis of poster-operative infection by modulating the signaling pathway of miR125b/STAT3/PCT.

Running title: Dysregulation of miRNA-125b is involved in post-operative infection

Key words: PCT, miRNA-125b, procalcitonin, post-operative infection

\section{Introduction}

Infections occurring after operation are a type of inflammation and adaptive response induced by noxious conditions and stimuli, including tissue injury and infection [1]. Scholars have made remarkable progress in clarifying the molecular and cellular events related to acute inflammatory responses not only to infection but also, to a lower extent, to the injury of tissues. Furthermore, the events causing local chronic inflammation, especially in autoimmune diseases and chronic infections, are partially understood [1].

The peptide PCT comprises 116 amino acids. Calcitonin (CT) is a hormone produced by C cells of thyroids and is related to the homeostasis of calcium. As a precursor of CT, PCT expression is generally undetectable in healthy people. A study on 78 healthy people indicated that the concentration of PCT with respect to both males and females is lower than $0.04 \mathrm{ng} / \mathrm{ml}$ [2]. The peptide PCT has become an early indicator specific and sensitive for bacterial infection in the last ten years $[3,4]$. Nevertheless, recently, studies indicated that the levels of PCT were also enhanced in clinical conditions without infections, including in major surgery, shock, burns, trauma, massive blood transfusion or cardiopulmonary bypass (CPB) [5-7]. 
STAT3 modulates the response of the host to the infection of bacteria for maintaining the function of the epithelial barrier of the gut through controlling the growth of bacteria and inhibiting apoptosis. According to Wittkopf et al., mice with conditional knockout of intestinal epithelial STAT3 (STAT3 IIEC) develop serious colitis and confer hypersusceptibility to infections in stomachs and gut [8]. Prior researches have confirmed that after the stimulation of lipopolysaccharide (LPS), increased levels of the protein STAT3 and activated STAT3 gene have been indicated to cause enhanced expression levels of PCT, and the levels of the expression of PCT occurred through the regulation of phosphoStat3 [9]

Recently, miRNAs have been discovered as a new type of short endogenous singlestranded RNA molecules (about 22 nucleotides). In 1993, miRNAs were found in the nematode Caenorhabditis elegans for the first time, but mammalian miRNAs were only discovered 12 years ago [10]. MiRNAs are conserved in humans and animals, and may possibly modulate $30 \%$ of all the genes in human genome [11]. STAT3 belongs to transcription factors and could regulate the invasion and proliferation of different cancer cells [12]. Furthermore, miR-21 and let-7a regulated STAT3 signaling of cancer cells of human cervices upon the induction of papillomavirus [13]. Moreover, STAT3 could be directly targeted by miR-125b in cancer cells of cervices and hence the silencing of STAT3 was able to decrease cervical cancer progression. Furthermore, in SiHa and HeLa cells, SNHG12 was able to modulate the expression level of STAT3 through sponging miR-125b. In summary, SNHG12 is potentially a new biomarker for diagnosing or treating cervical cancer and hence it was implicated that SNHG12/miR-125b/STAT3 axis is related to cervical cancer progression.

PCT has been reported to function as a diagnostic biomarker in the management of postoperative infection $[14,15]$. Furthermore, several miRNAs, including miR-125b, miR-20a and miR-106b, may act as negative regulators of PCT [16-18]. In this study, we enrolled subjects with or without post-operative infection to study the effects of miR-125b, miR$20 \mathrm{a}$ and miR-106b on the diagnosis of post-operative infection, so as to establish the role of the miR-125b/STAT3/PCT signaling pathway in post-operative infection. 


\section{Materials and Methods}

\section{Human sample collection}

A total of 130 children undergoing pediatric surgeries were enrolled in our research. These patients included 72 children diagnosed with post-operative infection (infection group) and 58 children without post-operative infection (non-infection group). The types of the undergoing pediatric surgeries of both groups were specified in Table 1. Peripheral blood samples were collected from all subjects at days 1, 2, 3, 4, and 5 after the surgery to test the levels of PCT, CRP, ESR and miR-125b expression in peripheral blood samples. At the same time, the clinicopathological and demographic characteristics of the participants, including age, sex, body weight, aspartate aminotransferase, creatinine, blood urea nitrogen, alanine aminotransferase, mechanical ventilation duration, length of PICU stay and mortality, were also collected and summarized. All procedures were approved by the institutional ethics committee and written informed consents were obtained before the initiation of this study.

\section{Biologic Measurement}

Blood samples used for the determination of concentrations of white blood cell (WBC), Creactive protein (CRP) and PCT in peripheral blood were collected separately following anesthesia induction (baseline) and then daily till the 5th postoperative day (POD). PCT was determined through an immunoluminometric assay of which the detection limit ranged from 0.05 to $25 \mathrm{ng} / \mathrm{mL}$ and the normal value was lower than $0.5 \mathrm{ng} / \mathrm{mL}$. CRP was determined through automatic laser nephelometry and the normal value was lower than $8 \mathrm{mg} / \mathrm{L}$. WBC was measured using an automatic counter and the normal value was between 4,000 and 10,000 cells $/ \mathrm{mm}^{3}$. Furthermore, the coefficient of variation of the measurements was lower than $5 \%$.

\section{Postoperative Infection Diagnosis and Grouping}

By definition, postoperative infections were as follows: 1) pneumonia: a) One of the symptoms as follows occurs: body temperature greater than $38^{\circ} \mathrm{C}$, auscultation findings 
suspicious of hypoxemia or pneumonia, purulent sputum; b) Leukocytosis (more than 12,000 cells $/ \mathrm{mm}^{3}$ ); c) Infiltrate according to chest radiograph; and d) Microorganism isolated from bronchial secretions. 2) Mediastinitis and deep sternal wound infection: a) Leukocytosis (> 12,000 cells $/ \mathrm{mm} 3$ ); b) Body temperature higher than $38^{\circ} \mathrm{C}$; and c) Presence of bacterial growth, pus, or both, found in samples of mediastinal tissues collected in surgical re-exploration. 3) Sepsis.

The final diagnosis of post-operation infection was determined by two experts independent of each other with respect to the complete medical chart. When the two experts did not agree with each other, a third expert was involved to reach a consensus. Each final diagnosis was grouped under "absent", "low probability (unlikely)", "possible", or "certain (high probability)". The final diagnosis was obtained when it was grouped under "possible" or "certain" by the above mentioned experts and ruled out when the experts grouped it under "unlikely" or "absent". Experts were blinded with respect to PCT but not for CRP and WBC, which were used routinely. There were two groups in the present study: the non-infection group involved people without the development of any postoperative infection within 5 days after the operation, and the infection group involved people with the development of postoperative infection within 5 days after surgery.

\section{Diagnosis of Noninfectious Complications}

Postoperative infection might also be accompanied with other noninfectious complications, which interfered with the level of PCT, so the authors also evaluated postoperation noninfectious complications. In the present study, by definition, post-operation noninfectious complications were as follows: 1) low cardiac output syndrome (LCOS) featured clinical symptoms and signs of low cardiac output (cardiac arrest, poor perfusion, tachycardia, and oliguria) requiring inotropic support equal to or higher than $100 \%$ above baseline, the administration of a new inotropic drug, or malignant ventricular arrhythmia, high output failure needing the use of delayed sternal closure/extracorporeal membrane oxygenation support or other maneuvers used to 
enhance cardiac output (i.e., cardiac pacing); 2) renal insufficiency needing continuous venovenous hemofiltration treatment or peritoneal dialysis; 3) gastrointestinal complication (ischemia, bleeding); and 4) neurologic complication (ischemic injury, stroke).

\section{Determination of inflammatory markers}

The levels of inflammatory markers were determined using a BD clinical chemistry analyzer (Fullerton, CA) with an error range of lower than $15 \%$.

\section{RNA isolation/real-time PCR}

Total RNA samples were isolated using an QIAamp Blood RNA Extraction Kit (Thermo Fisher Scientific, Waltham, MA) following kit instructions. The concentration of isolated RNA samples was measured using NanoDrop (Thermo Fisher Scientific, Waltham, MA). The reaction of reverse transcription was carried out using a cDNA RT Kit (Thermo Fisher Scientific, Waltham, MA). Finally, real-time PCR was carried out using a SYBR Green Master kit (Roche, OR, USA) on a LightCycler real-time PCR machine to quantify the relative expression of miR-125b (Forward primer: 5'-GGCAACCTTGCGACTATAACCA-3'; Reverse primer: 5'-GTTTCCTCTCCCTGAGACCCTA-3'), miR-106b (Forward primer: 5'TGGGACACTGGGTATACG-3'; Reverse primer: 5'-ACTCTACCGGATCACAGC-3'), miR-20a (Forward primer: 5'- CTCGTAAATGTTATGAATCC-3'; Reverse primer: 5'TCTCCAGTAGAAATAGCA-3'), and STAT3 mRNA (Forward primer: 5'CTTTGAGACCGAGGTGTATCACC-3'; Reverse primer: 5'- GGTCAGCATGTTGTACCACAGG-3').

\section{Cell culture and treatment}

THP-1 and U937 cells were obtained from ATCC and maintained in DMEM (Invitrogen, Carlsbad, CA) with $10 \% \mathrm{FBS}$. The cell culture was done in a $37{ }^{\circ} \mathrm{C}$ incubator set to $5 \% \mathrm{CO}_{2}$ and $95 \%$ air. When the cells were confluent, they were seeded into 96 -well plates at $1 \times$ $10^{4}$ cells/well for overnight culture. On the second day, the cells were transfected with miR-125b, miR-20a or miR-106b for $48 \mathrm{~h}$ using Lipofectamine 2000 (Invitrogen, Carlsbad, CA) according to the standard protocol provided by the manufacturer. Upon the 
completion of transfection, the cells were harvested to measure their expression of miR125b, miR-20a or miR-106b. Similarly, THP-1 and U937 cells were cultured in 96-well plates and treated with a negative control (NC), LPS, LPS + miR-125b, miR-125b inhibitor, and LPS + miR-125b inhibitor for 2 days before the expression of various target genes was analyzed.

\section{Luciferase assay}

The locations of miR-125b, miR-106b and miR-20a binding sites in the 3'UTR of STAT3 was determined using bioinformatics tools. Subsequently, the 3'UTR fragments of STAT3 containing the miR-125b, miR-106b and miR-20a binding sites were respectively cloned into pcDNA vectors (Promega, Madison, WI) to generate wild type vectors of STAT3 3'UTR for each miRNA. Then, site-directed mutagenesis was carried out at the miR-125b, miR$106 \mathrm{~b}$ and miR-20a binding sites of STAT3 3'UT, respectively, to create mutant vectors of STAT3 3'UTR for each miRNA. Finally, THP-1 and U937 cells were cultured in 96-well plates and treated for $48 \mathrm{~h}$ with wild type or mutant vectors of STAT3 3'UTR in conjunction with miR-125b mimics, miR-106b mimics or miR-20a mimics. Upon the completion of cell treatment, the luciferase activity of transfected cells was measured using a dual luciferase assay (Promega, Madison, WI).

\section{Western blot analysis}

Samples were homogenized in an appropriate volume of lysis buffer (Sigma-Aldrich, St. Louis, MO) at $4{ }^{\circ} \mathrm{C}$ for $30 \mathrm{~min}$ and centrifuged at $4{ }^{\circ} \mathrm{C}$ and $15,000 \times g$ for 10 to collect the supernatant. The protein samples in the supernatant were adjusted to the same concentration and were resolved by $15 \%$ SDS-PAGE. Subsequently, the resolved protein bands were blotted onto a PVDF membrane, which was then blocked at room temperature for 60 min using 5\% skim milk, rinsed with a TBST buffer, incubated overnight at $4{ }^{\circ} \mathrm{C}$ with primary anti-STAT3 (cat.no: 9139S, Cell Signaling Technology, Danvers, MA, US) and anti-p-STAT3 antibodies (cat.no: 9145S, Cell Signaling Technology, Danvers, MA, US) under constant shaking, rinsed again with the TBST buffer, incubated at room temperature for $60 \mathrm{~min}$ with secondary IgG antibodies labeled by horseradish 
peroxidase (cat.no: 7074S, Cell Signaling Technology, Danvers, MA, US), and developed using an ECL reagent (Sigma-Aldrich, St. Louis, MO) to determine the relative protein expression of STAT3 and p-STAT3 with Image J software.

\section{Statistical analysis}

SPSS version 16.0 was used to compare the above characteristics between the two groups using Student t test. Comparisons between multiple groups were performed via one way ANOVA, with Scheffe's test as the post hoc test. An analysis of Receiver Operating Characteristic (ROC) was carried out to determine the AUC values of PCT, CRP, ESR, miR125b, miR-106b and miR-20a, and whether they were associated with post-operative infection. All data was expressed as mean \pm standard deviation, while $\mathrm{P}<0.05$ was used as the threshold for statistical significance. Each experiment was repeated in triplicate.

\section{Results}

\section{Characteristics of the participants with or without post-operative infection}

We recruited 130 children who underwent pediatric surgeries in our research. Among them, 72 children were diagnosed with post-operative infection and 58 children were diagnosed without post-operative infection. As demonstrated in Table 1, demographic and clinicopathological characteristics of the participants exhibited no obvious difference between these two groups.

\section{MiR-125b directly targeted STAT3}

Computational software via an online database of miRNA (www.mirdb.org) was utilized to search potential targeting miRNAs of STAT3, and putative binding sites of miR-125b (Fig. 1A), miR-106b (Fig. 1B) and miR-20a (Fig. 1C) were identified on STAT3 3'UTR. Luciferase assays were then conducted to confirm whether these miRNA binding sites were functional. An evident decrease in the luciferase activity of wild-type STAT3 3'UTR but not that of mutant STAT3 3'UTR was observed in THP-1 and U937 cells (Fig. 1A) transfected with miR-125b mimics. However, both miR-106a mimics (Fig. 1B) and miR20a (Fig. 1C) mimics showed no effect on the luciferase activity of wild-type or mutant 
STAT3 3'UTR in THP-1 and U937 cells, validating that STAT3 was a virtual target gene of miR-125b only.

\section{PCT might be used as a diagnostic biomarker of post-operative infection}

PCT, CRP and ESR were previously described as markers for infection. In this study, blood samples were collected preoperatively (day 0) and post-operatively (days 1, 2, 3, 4 and 5) to evaluate the values of PCT, CRP and ESR as predictive biomarkers of post-operative infection. As shown in Fig. 2A and 2B, the PCT level in the non-infection group (Fig. 2A) showed no obvious difference from day 0 to day 5, while the PCT level in the infection group (Fig. 2B) was evidently increased from day 1 to day 5 in a time-dependent manner. In addition, the post-operative levels of CRP (Fig. 2C and 2D) and ESR (Fig. 2E and 2F) in both non-infection (Fig. 2C and 2E) and infection (Fig. 2D and 2F) groups were evidently increased in a time-dependent manner. Therefore, only PCT might serve as a predictive and diagnostic biomarker of post-operative infection.

\section{MiR-125b and PCT were involved in post-operative infection}

Real-time PCR was performed to compare the expression of miR-125b, miR-106b and miR-20a between non-infection and infection groups from day 0 to day 5 . As shown in Fig. 3, the miR-125b level in the non-infection group (Fig. 3A) showed no obvious difference from day 0 to day 5, while the miR-125b level in the infection group (Fig. 3B) was significantly down-regulated from day 1 to day 5 in a time-dependent manner. In addition, the post-operative expression of both miR-106b (Fig. 3C and 3D) and miR-20a (Fig. 3E and 3F) was remained stable in both non-infection (Fig. 3C and 3E) and infection (Fig. 3D and 3F) groups. This might be contributed to the fact that the inflammatory biomarkers last in infection group, while they fade away in non-infection group. Meanwhile, an analysis of Receiver Operating Characteristic (ROC) was carried out to determine whether PCT, CRP, ESR, miR-125b, miR-106b and miR-20a were associated with post-operative infection. As shown in Fig. 4, the AUC values of PCT, CRP, ESR, miR-125b, miR-106b and miR-20a were $0.75,0.55,0.55,0.85,0.5$ and 0.7 respectively, demonstrating that miR-125b and PCT were involved in post-operative infection. 


\section{MiR-125b reduced STAT3 expression}

The mRNA and protein levels of STAT3 were detected in THP-1 and U937 cells transfected with miR-125b mimics, miR-106b mimics or miR-20a mimics using real-time PCR and Western-blot analysis. As shown in Fig. 5, only the transfection with miR-125b mimics reduced the mRNA and protein levels of STAT3 in THP-1 (Fig. 5A) and U937 (Fig. 5B) cells.

\section{Activation of STAT3 increased PCT production}

To explore the mechanism through which miR-125b affected PCT expression, we carried out a bioinformatics analysis on LPS-related pathways. After screening the expression of MAPK13, TLR4 and STAT3, STAT3 was selected for further study. The phosphorylation of STAT3 in THP-1 (Fig. 6A) and U937 (Fig. 6E) cells was substantially elevated following LPS treatment. The up-regulation of STAT3 in THP-1 (Fig. 6B) and U937 (Fig. 6F) cells facilitated the phosphorylation of STAT3 and promoted PCT expression. On the contrary, STAT3 shRNAs repressed the phosphorylation of STAT3 (Fig. 6C and 6G) and notably reduced the PCT level in THP-1 (Fig. 6D) and U937 (Fig. 6H) cells.

\section{MiR-125b inhibited PCT expression by targeting STAT3}

STAT3 was proved as a target gene of miR-125b. To further investigate the potential role of miR-125b in the regulation of PCT expression, we examined LPS-induced activation of STAT3 in the presence or absence of miR-125b. The transfection of STAT3 in THP-1 (Fig. 7A) and U937 (Fig. 7G) cells significantly decreased the level of phosphorylated STAT3 with the transfection of miR-125b inhibitors. In addition, the mRNA level of PCT in THP-1 (Fig. 7C) and U937 (Fig. 7l) cells was moderately increased by miR-125b inhibitors but significantly increased by STAT3 (Fig. 7B and 7G). Furthermore, the inhibition of miR-125b significantly increased the level of total STAT3 but only slightly increased the level of phospho-STAT3 (Fig. 5C and H), while the inhibition of miR-125b significantly up-regulated PCT production in THP-1 (Fig. 5D) and U937 (Fig. 5I) cells. The DNA-binding activity of STAT3 was significantly up-regulated after transfection with miR-125b inhibitors (Fig. 5E and J). 


\section{Discussion}

The finding of this present study verified our hypothesis that several miRNAs miR-125b, miR-20a and miR-106b participated in the molecular signaling pathway underlying the diagnosis of post-operative infection by PCT. In this study, we demonstrated that only PCT could be used as a predictive biomarker of post-operative infection, and we also confirmed that only the level of miR-125a was associated with the progression of postoperative infection. Meanwhile, by performing an ROC analysis, we found that only miR$125 \mathrm{~b}$ and PCT were involved in post-operative infection. Previously, researches have exhibited that the treatment with LPS for the PBMs enhanced the PCT level and decreased the miR-125b level [19]. In addition, the treatment with LPS enhanced the expression of total STAT3 and phosphorylated STAT3, which in turn increased the expression level of PCT [19]. Nevertheless, previously, it has been indicated that miR-125b and the lincRNA of the gene of NED25 increased the levels of total STAT3 and phosphorylated STAT3 while lowering the expression level of PCT [20].

MicroRNA-125b (miR-125b) is a lin-4 homolog in mammals, and lin-4 is the miRNA first identified in Caenorhabditis elegans [21] Furthermore, miR-125b is related to the development of immune systems and the immunological defense of the host. In macrophages, overexpressed miR-125b enables the function of macrophages for immune responses and enhances the responsiveness to IFN-g [22]. The expression of Hepatitis B virus is suppressed by miR-125b according to cell culture trials [23]. VirulentMycobacterium tuberculosis-produced lipomannan inhibits the biosynthesis of TNF via miR-125b induction, which targets the 3' UTR region of the mRNA of TNF and makes the transcript unstable [24]. Previously, studies indicated that many miRNAs involved in the pathway of Salmonella infection and miR-27b, miR-125b and miR-221 played a significant role. It was also shown in myelopoiesis that miR-125b affected the activity of the transcription of STAT3 [25]. Previously, STAT3 was predicted as a candidate target gene of miR-125b, and subsequent luciferase assay also confirmed that the activity of luciferase of the wild-type STAT3 3'UTR was suppressed when miR-125b was present [26]. In this 
study, we also identified STAT3 as a direct target of miR-125b, and the over-expression of miR-125b could reduce the expression of STAT3.

In inflammation, STAT3 plays a dual self-perpetuating role. With respect to infection, at early stages, the activation of STAT3 facilitated inflammation; while at later stages, the sustained activation of STAT3 inhibited inflammation and facilitated proliferation 122. Previously, researches also indicated that STAT3 played various roles in colon cancer progression. With respect to infection, during early infection (<20 weeks) of DSS mice, the activation of STAT3 possibly promotes inflammation, while during later infection (45 weeks), the sustained activation of STAT3 facilitated proliferation of cancer cells and inhibited inflammatory responses [27].

PCT and STAT3 were the lowest in the control group of healthy people and were the highest in the group of septic shock patients. Generally, LPS or other bacteria-secreted toxins can directly induce PCT release during inflammation [28]. Nevertheless, the cellular response to inflammation-related cytokines can also indirectly or directly induce PCT release [29]. Previously, researches have confirmed that miR-125b in monocytes regulates the level of PCT through mediating the transcription of STAT3 [16]. In this study, we validated that STAT3 could increase PCT synthesis. Moreover, after assessing the effect of LPS-induced STAT3 activation on the treatment of post-operative infection with or without the presence of miR-125b mimics/inhibitors, we came to the conclusion that miR-125b could inhibit PCT expression by targeting STAT3.

Initially identified as a calcitonin prohormone, the 12.6-kDa polypeptide procalcitonin (PCT) contains 114 amino acids and is related to the metabolism of calcium. PCT is produced by medullary C-cells in thyroids [30-32]. Nevertheless, recently, researches have confirmed that variant PCT is able to be generated in other tissues upon infection [32-34]. Dependent on the severity and prognosis of sepsis, PCT levels are gradually enhanced in systemic inflammation [35]. Thus, the continuous determination of PCT levels in the intensive care unit (ICU) may possibly facilitate the early use of antibiotics [36]. Furthermore, using a PCT value of $<0.6 \mathrm{ng} / \mathrm{mL}$ as the threshold for its minimum 
reduction, some researchers failed to carry out an accurate prediction of treatment responses in septic shock patients [37]. The use of PCT as an indicator at early stages of infections in children, infants, and neonates produced adequate specificity and sensitivity. Therefore, PCT may be considered to be an additional method to identify bacterial infections for such patients. Other than its favorable specificity and sensitivity, PCT has another advantage for clinical practice: its correlation with the seriousness of infection is high [38].

One novelty of our study was that we observed the dynamic changes of inflammatory indicators of the recruited patients. And apart from the inflammatory indicators, we also explored the up-stream molecular signaling pathway underlying the pathogenesis of this study. However, there are limitations of our study. The sample size is limited, and the parameters we studied were also limited. In the future, more comprehensive study is warranted.

\section{Conclusion}

In conclusion, this study demonstrated that miRNA-125b was involved in the prognosis of post-operative infection by modulating the signaling pathways downstream of STAT3 as well as the procalcitonin (PCT) pathway.

\section{Conflict of interest}

None

\section{Acknowledgment:}

We sincerely appreciate the support from central laboratory of Fujian Medical University Union Hospital.

\section{Availability of data and material}

The data that support the findings of this study are available from the corresponding author upon reasonable request.

\section{Author contributions}


XJ and LW planned the study, collected the literatures, LW and MZY collected and analyzed the data, MZY and XJ composed the manuscript, and all the other co-authors approved the final manuscript.

\section{Figure legends}

\section{Table 1}

Characteristics of the subjects enrolled in this study

\section{Figure 1}

STAT3 was a candidate target gene of miR-125b

A: MiR-125b directly targeted its "seed sequence" in the 3'-UTR of STAT3, and miR-125b reduced the luciferase activity of wild-type STAT3 $3^{\prime}$ UTR in THP-1 and U937 cells $\left({ }^{*} \mathrm{P}<\right.$ 0.05 , vs. control group)

B: MiR-106b directly targeted its "seed sequence" in the 3'-UTR of STAT3, but miR-106b had no effect on the luciferase activity of wild-type or mutant STAT3 3'UTR in THP-1 and U937 cells

C: MiR-20a directly targeted its "seed sequence" in the 3'-UTR of STAT3, but miR-20a had no effect on the luciferase activity of wild-type or mutant STAT3 3'UTR in THP-1 and U937 cells

\section{Figure 2}

PCT might act as a predictive and diagnostic biomarker of post-operative infection

A: PCT expression in non-infection group showed no obvious difference from day 0 to day 5

B: PCT expression in infection group was evidently increased from day 1 to day 5 after surgery in a time-dependent manner

C: CRP expression in non-infection group was evidently increased from day 1 to day 5 after surgery in a time-dependent manner 
D: CRP expression in infection group was evidently increased from day 1 to day 5 after surgery in a time-dependent manner

E: Level of ESR in non-infection group was significantly up-regulated from day 1 to day 5 after surgery in a time-dependent manner

F: Level of ESR in infection group was significantly up-regulated from day 1 to day 5 after surgery in a time-dependent manner

\section{Figure 3}

miR-125b was involved in post-operative infection

A: miR-125b level in non-infection group showed no obvious difference from day 0 to day 5

B: miR-125b level in infection group was evidently decreased from day 1 to day 5 after surgery in a time-dependent manner

C: miR-106b level in non-infection group showed no obvious difference from day 0 to day 5

D: miR-106b level in infection group showed no obvious difference from day 0 to day 5

E: Level of miR-20a in non-infection group remained stable from day 0 to day 5

F: Level of miR-20a in infection group remained stable from day 0 to day 5

\section{Figure 4}

MiR-125b and PCT were associated with post-operative infection

A: The AUC values of PCT, CRP, and ESR were $0.75,0.55$ and 0.55 , respectively

B: The AUC values of miR-125b, miR-106b and miR-20a were 0.85, 0.5 and 0.7, respectively

\section{Figure 5}


MiR-125b reduced STAT3 expression

A: The mRNA and protein levels of STAT3 in THP-1 cells was significantly reduced by the transfection with miR-125b mimics, while miR-106b and miR-20a mimics had no effect on STAT3 expression (* P value $<0.05$, vs. NC group; NC: negative control).

B: The mRNA and protein levels of STAT3 in U937 cells was significantly reduced by the transfection with miR-125b mimics, while miR-106b and miR-20a mimics had no effect on STAT3 expression (* P value $<0.05$, vs. NC group; NC: negative control).

\section{Figure 6}

Activation of STAT3 increased PCT production

A: Levels of total STAT3 and phospho-STAT3 in THP-1 cells were increased by LPS treatment and further up-regulated by LPS + STAT3 treatment

B: PCT production in THP-1 cells was increased by LPS treatment and further up-regulated by LPS + STAT3 treatment (* P value $<0.05$, vs.Stat3 (-) LPS (-) group)

C: LPS-induced production of total STAT3 and phosphorylated STAT3 was repressed in THP-1 cells by shRNA-mediated STAT3 knockdown

D: ShRNA-mediated STAT3 knockdown repressed LPS-induced production of PCT in THP1 cells (* P value $<0.05$, vs.Stat3 (-) LPS (-) group; ** P value $<0.05$, vs. LPS (+) Stat3 (-) group)

E: Levels of total STAT3 and phospho-STAT3 in U937 cells were increased by LPS treatment and further up-regulated by LPS + STAT3 treatment

F: PCT production in U937 cells was increased by LPS treatment and further up-regulated by LPS + STAT3 treatment (* P value $<0.05$, vs.Stat3 (-) LPS (-) group)

G: LPS-induced production of total STAT3 and phosphorylated STAT3 was repressed in U937 cells by shRNA-mediated STAT3 knockdown 
H: ShRNA-mediated STAT3 knockdown repressed LPS-induced production of PCT in U937 cells (* P value $<0.05$, vs.Stat3 (-) LPS (-) group; ${ }^{* *}$ P value $<0.05$, vs. LPS (+) Stat3 (-) group)

\section{Figure 7}

MiR-125b inhibited PCT expression by targeting STAT3

A: Levels of total STAT3 and phospho-STAT3 in THP-1 cells were decreased by miR-125b but increased by STAT3 treatment

B: LPS-induced production of phosphorylated STAT3 but not total STAT3 was repressed in THP-1 cells by miR-125b inhibitors but increased by STAT3 treatment

C: PCT production in THP-1 cells was decreased by miR-125b but increased by STAT3 treatment (*P value $<0.05$, vs. miR-125 inhibitors (-) LSP (-) group; ** P value $<0.05$, vs. miR-125 inhibitors(-) LSP (+) group)

D: Inhibition of miR-125b increased the level of PCT in THP-1 cells (* P value $<0.05$, vs. miR-125 inhibitors (-) Stat3 (-) LSP (-) group; ** P value $<0.05$, vs. miR-125 inhibitors(-) Stat3 (-) LSP (+) group)

E: DNA-binding activity of STAT3 was obviously increased in THP-1 cells by miR-125b inhibitors

F: Levels of total STAT3 and phospho-STAT3 in THP-1 cells were decreased by miR-125b but increased by STAT3 treatment

G: PCT production in THP-1 cells was decreased by miR-125b but increased by STAT3 treatment

H: LPS-induced production of phosphorylated STAT3 but not total STAT3 was repressed in THP-1 cells by miR-125b inhibitors but increased by STAT3 treatment 
I: Inhibition of miR-125b increased the level of PCT in THP-1 cells (* P value $<0.05$, vs. miR-125 inhibitors (-) LSP (-) group; ** P value < 0.05, vs. miR-125 inhibitors(-) LSP (+) group)

J: DNA-binding activity of STAT3 was obviously increased in THP-1 cells by miR-125b inhibitors

\section{References}

1 Medzhitov R: Origin and physiological roles of inflammation. Nature 2008;454:428-435.

2 Giovanella L: Serum procalcitonin and calcitonin normal values before and after calcium gluconate infusion. Exp Clin Endocrinol Diabetes 2012;120:169-170.

3 Angeletti S, Spoto S, Fogolari M, Cortigiani M, Fioravanti M, De Florio L, Curcio B, Cavalieri D, Costantino S, Dicuonzo G: Diagnostic and prognostic role of procalcitonin (PCT) and MR-pro-Adrenomedullin (MR-proADM) in bacterial infections. APMIS 2015;123(9):740-748.

4 Mohan A, Harikrishna J: Biomarkers for the diagnosis of bacterial infections: in pursuit of the 'Holy Grail'. Indian J Med Res 2015;141(3):271.

5 Fritz HG, Brandes H, Bredle DL, Bitterlich A, Vollandt R, Specht M, Franke UF, Wahlers T, Meier-Hellmann A: Post-operative hypoalbuminaemia and procalcitonin elevation for prediction of outcome in cardiopulmonary bypass surgery. Acta Anaesthesiol Scand 2003;47(10):1276-1283..

6 Balci C, Sivaci R, Akbulut G, Karabekir HS: Procalcitonin Levels as an Early Marker in Patients with Multiple Trauma under Intensive Care. J Int Med Res 2009; 37(6):1709-1717..

7 Ruiz-Rodríguez JC, Caballero J, Ruiz-Sanmartin A, Ribas VJ, Pérez M, Bóveda JL, Rello J: Usefulness of procalcitonin clearance as a prognostic biomarker in septic shock. A prospective pilot study. Med Intensiva 2012;36(7):475-480. 
8 Wittkopf N, Pickert G, Billmeier U, Mahapatro M, Wirtz S, Martini E, Leppkes M, Neurath MF, Becker C: Activation of intestinal epithelial Stat3 orchestrates tissue defense during gastrointestinal infection. PLoS One 2015;10:e0118401.

9 Becker KL, Snider R, Nylen ES: Procalcitonin in sepsis and systemic inflammation: a harmful biomarker and a therapeutic target. Br J Pharmacol 2010;159:253-264.

10 Lagos-Quintana M, Rauhut R, Lendeckel W, Tuschl T: Identification of novel genes coding for small expressed RNAs. Science 2001;294:853-858.

11 Lewis BP, Burge CB, Bartel DP: Conserved seed pairing, often flanked by adenosines, indicates that thousands of human genes are microRNA targets. Cell 2005;120:15-20.

12 Yuan J, Zhang F, Niu R: Multiple regulation pathways and pivotal biological functions of STAT3 in cancer. Sci Rep 2015;5:17663.

13 Shishodia G, Verma G, Srivastava Y, Mehrotra R, Das BC, Bharti AC: Deregulation of microRNAs Let-7a and miR-21 mediate aberrant STAT3 signaling during human papillomavirus-induced cervical carcinogenesis: role of E6 oncoprotein. BMC Cancer 2014;14:996.

14 Li X, Wang X, Li S, Yan J, Li D: Diagnostic Value of Procalcitonin on Early Postoperative Infection After Pediatric Cardiac Surgery. Pediatr Crit Care Med 2017;18:420-428.

15 Aljabi Y, Manca A, Ryan J, Elshawarby A: Value of procalcitonin as a marker of surgical site infection following spinal surgery. Surgeon 2019;17:97-101.

16 Zhang F, Fan X, Bai Y, Lu J, Zheng M, Chen J, Liu Y, Chen Z, Zhu J: miR-125b regulates procalcitonin production in monocytes by targeting Stat3. Microbes Infect 2016;18:102-108.

17 He Z, Jiang J, Kokkinaki M, Tang L, Zeng W, Gallicano I, Dobrinski I, Dym M: MiRNA20 and mirna-106a regulate spermatogonial stem cell renewal at the posttranscriptional level via targeting STAT3 and Ccnd1. Stem Cells 2013;31:2205-2217. 
18 Liu X, Su X, Xu S, Wang H, Han D, Li J, Huang M, Cao X: MicroRNA in vivo precipitation identifies miR-151-3p as a computational unpredictable miRNA to target Stat3 and inhibits innate IL-6 production. Cell Mol Immunol 2018;15:99-110.

19 Le $\mathrm{Y}$, Chen T, Xun K, Ding T: Expression of the Long Intergenic Non-Coding RNA (lincRNA) of the NED25 Gene Modulates the microRNA-125b, STAT3, Nitric Oxide, and Procalcitonin Signaling Pathways in Patients with Sepsis. Med Sci Monit 2018;24:4555-4566.

20 Hua S, Liu X, Lv S, Wang Z: Protective Effects of Cucurbitacin B on Acute Lung Injury Induced by Sepsis in Rats. Med Sci Monit 2017;23:1355-1362.

21 Lee YS, Kim HK, Chung S, Kim KS, Dutta A: Depletion of human micro-RNA miR$125 \mathrm{~b}$ reveals that it is critical for the proliferation of differentiated cells but not for the down-regulation of putative targets during differentiation. J Biol Chem 2005;280:16635-16641.

22 Chaudhuri AA, So AY, Sinha N, Gibson WS, Taganov KD, O'Connell RM, Baltimore D: MicroRNA-125b potentiates macrophage activation. J Immunol 2011;187:50625068.

23 Zhang Z, Chen J, He Y, Zhan X, Zhao R, Huang Y, Xu H, Zhu Z, Liu Q: miR-125b inhibits hepatitis B virus expression in vitro through targeting of the SCNN1A gene. Arch Virol 2014;159:3335-3343.

24 Rajaram MV, Ni B, Morris JD, Brooks MN, Carlson TK, Bakthavachalu B, Schoenberg DR, Torrelles JB, Schlesinger LS: Mycobacterium tuberculosis lipomannan blocks TNF biosynthesis by regulating macrophage MAPK-activated protein kinase 2 (MK2) and microRNA miR-125b. Proc Natl Acad Sci U S A 2011;108:17408-17413.

25 Wu D, Zhou Y, Pan H, Zhou J, Fan Y, Qu P: microRNA-99a inhibiting cell proliferation, migration and invasion by targeting fibroblast growth factor receptor 3 in bladder cancer. Oncol Lett 2014;7:1219-1224.

26 Feng J, Fan Y, Ayiheng Q, Zhang H, Yong J, Hu B: MicroRNA-125b targeted STAT3 to inhibit laryngeal squamous cell carcinoma cell growth and motility. Oncol Lett 2017;14:480-486. 
27 Lu R, Zhang YG, Sun J: STAT3 activation in infection and infection-associated cancer. Mol Cell Endocrinol 2017;451:80-87.

28 Oberhoffer M, Stonans I, Russwurm S, Stonane E, Vogelsang H, Junker U, Jager L, Reinhart K: Procalcitonin expression in human peripheral blood mononuclear cells and its modulation by lipopolysaccharides and sepsis-related cytokines in vitro. J Lab Clin Med 1999;134:49-55.

29 Nijsten MW, Olinga P, The TH, de Vries EG, Koops HS, Groothuis GM, Limburg PC, ten Duis HJ, Moshage H, Hoekstra HJ, Bijzet J, Zwaveling JH: Procalcitonin behaves as a fast responding acute phase protein in vivo and in vitro. Crit Care Med 2000;28:458-461.

30 Weglohner W, Struck J, Fischer-Schulz C, Morgenthaler NG, Otto A, Bohuon C, Bergmann A: Isolation and characterization of serum procalcitonin from patients with sepsis. Peptides 2001;22:2099-2103.

31 Birnbaum RS, Mahoney WC, Burns DM, O'Neil JA, Miller RE, Roos BA: Identification of procalcitonin in a rat medullary thyroid carcinoma cell line. J Biol Chem 1984;259:2870-2874.

32 Jacobs JW, Lund PK, Potts JT, Jr., Bell NH, Habener JF: Procalcitonin is a glycoprotein. J Biol Chem 1981;256:2803-2807.

33 Ittner L, Born W, Rau B, Steinbach G, Fischer JA: Circulating procalcitonin and cleavage products in septicaemia compared with medullary thyroid carcinoma. Eur J Endocrinol 2002;147:727-731.

34 Meisner M, Muller V, Khakpour Z, Toegel E, Redl H: Induction of procalcitonin and proinflammatory cytokines in an anhepatic baboon endotoxin shock model. Shock 2003;19:187-190.

35 Jensen JU, Heslet L, Jensen TH, Espersen K, Steffensen P, Tvede M: Procalcitonin increase in early identification of critically ill patients at high risk of mortality. Crit Care Med 2006;34:2596-2602. 
36 Nobre V, Harbarth S, Graf JD, Rohner P, Pugin J: Use of procalcitonin to shorten antibiotic treatment duration in septic patients: a randomized trial. Am J Respir Crit Care Med 2008;177:498-505.

37 Jung B, Molinari N, Nasri M, Hajjej Z, Chanques G, Jean-Pierre H, Panaro F, Jaber S: Procalcitonin biomarker kinetics fails to predict treatment response in perioperative abdominal infection with septic shock. Crit Care 2013;17:R255.

38 Memar MY, Varshochi M, Shokouhi B, Asgharzadeh M, Kafil HS: Procalcitonin: The marker of pediatric bacterial infection. Biomed Pharmacother 2017;96:936-943. 


\begin{tabular}{|c|c|c|c|}
\hline Characteristics & Infection ( $N=72$ ) & Non-infection ( $\mathrm{N}=58$ ) & $P$ value \\
\hline Age & $8.5 \pm 4.3$ & $8.1 \pm 3.2$ & 0.621 \\
\hline Weight (kg) & $7.6 \pm 3.7$ & $8.6 \pm 2.4$ & 0.259 \\
\hline Male sex $(n, \%)$ & $42(58.3)$ & $36(62.1)$ & 0.523 \\
\hline $\begin{array}{l}\text { Aspartate aminotransferase } \\
(\mathrm{U} / \mathrm{L})\end{array}$ & $155.6 \pm 53.4$ & $154.4 \pm 53.8$ & 0.716 \\
\hline Alanine aminotransferase (U/L) & $28.5 \pm 8.8$ & $28.1 \pm 6.1$ & 0.869 \\
\hline Creatinine $(\mu \mathrm{mol} / \mathrm{L})$ & $44.8 \pm 11.5$ & $43.9 \pm 12.8$ & 0.483 \\
\hline Blood urea nitrogen (mmol/L) & $7.8 \pm 2.4$ & $8.1 \pm 3.5$ & 0.334 \\
\hline $\begin{array}{l}\text { Mechanical ventilation } \\
\text { duration (hr) }\end{array}$ & $24(6-133)$ & $19(5-57)$ & 0.322 \\
\hline PICU stay $(d)$ & $5(3-14)$ & $4(1-8)$ & 0.483 \\
\hline Death $(n, \%)$ & $1(1.4)$ & $1(1.7)$ & 0.785 \\
\hline Operation & & & 0.612 \\
\hline Atrial septal defect & 30 & 23 & \\
\hline Ventricular septal defect & 21 & 16 & \\
\hline Tetrology of fallot & 12 & 8 & \\
\hline Pulmoanry arterial atresia & 8 & 8 & \\
\hline Transposition of great arteries & 1 & 3 & \\
\hline
\end{tabular}

Table 1. Characteristics of the subjected enrolled in this study 
A
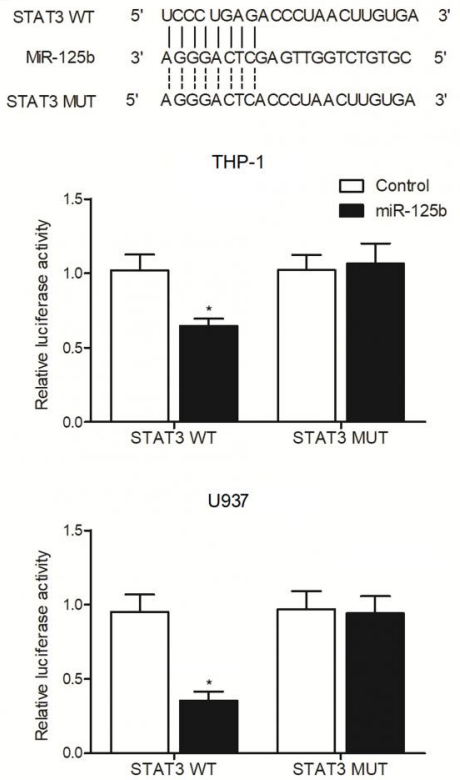

B

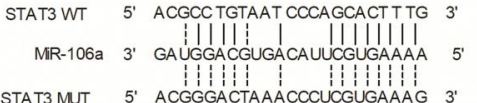

THP-1

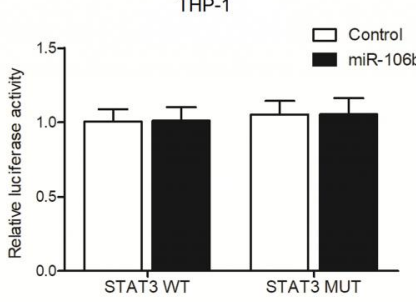

U937

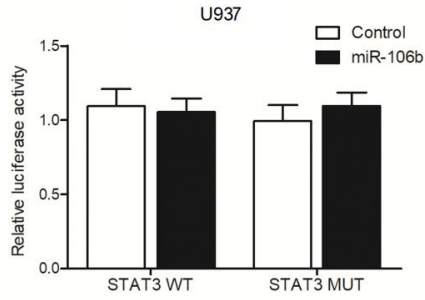

C

STAT3 WT 5' ACGCC TG TAATCCCAGCACT TT T

Mr-20a 3. GauggacGUgauauucgugaAau

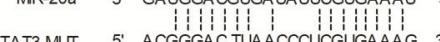

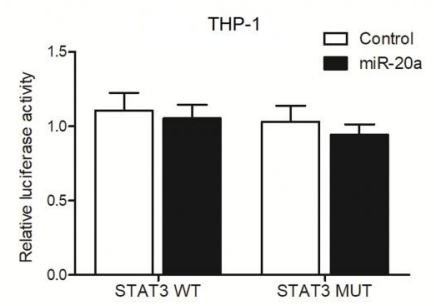

U937

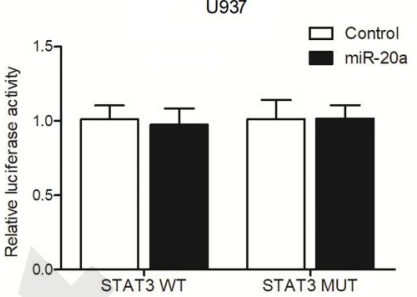

Figure 1

STAT3 was a candidate target gene of miR-125b

A: MiR-125b directly targeted its "seed sequence" in the 3'-UTR of STAT3, and miR-125b reduced the luciferase activity of wild-type STAT3 3'UTR in THP-1 and U937 cells ( ${ }^{*} \mathrm{P}<$ 0.05 , vs. control group)

B: MiR-106b directly targeted its "seed sequence" in the 3'-UTR of STAT3, but miR-106b had no effect on the luciferase activity of wild-type or mutant STAT3 3'UTR in THP-1 and U937 cells

C: MiR-20a directly targeted its "seed sequence" in the 3'-UTR of STAT3, but miR-20a had no effect on the luciferase activity of wild-type or mutant STAT3 3'UTR in THP-1 and U937 cells 
A

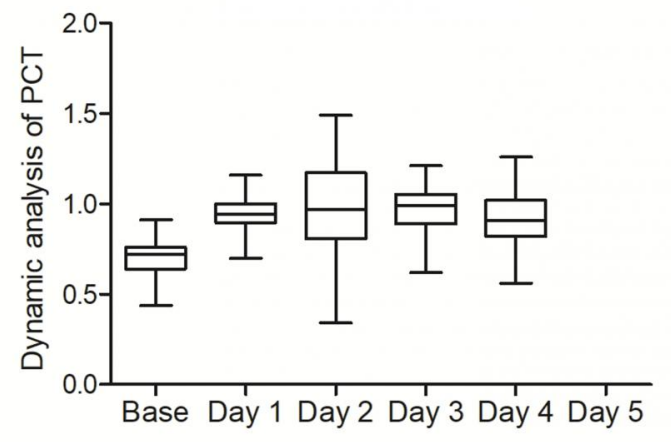

B

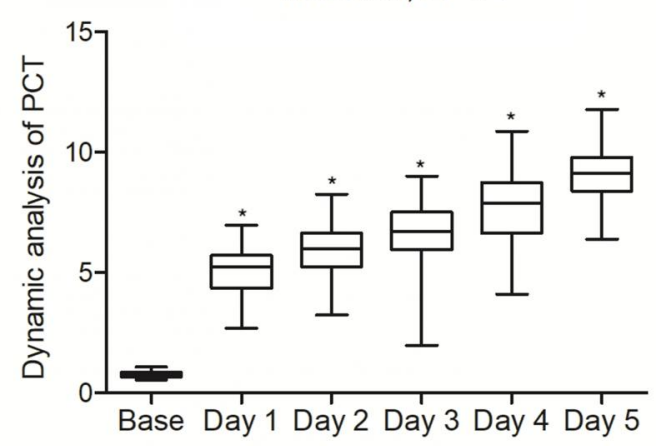

D Infection, $\mathrm{N}=72$
C

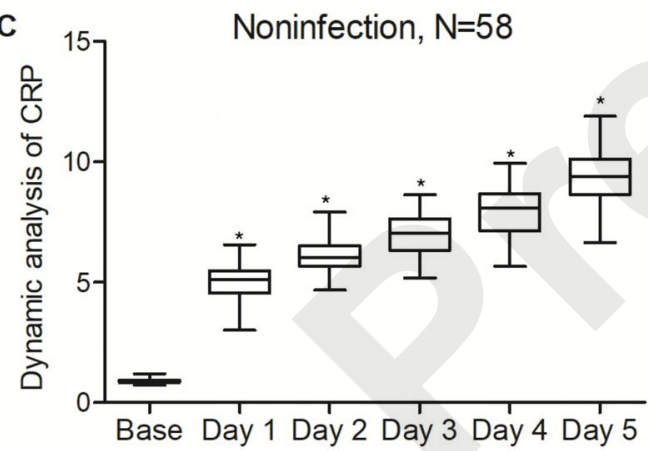

$E$

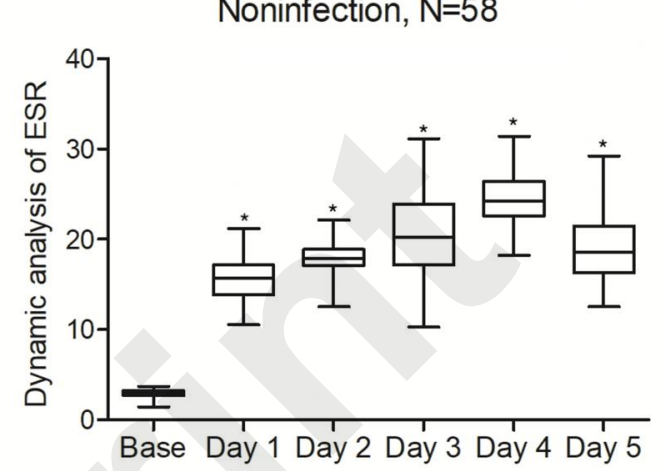

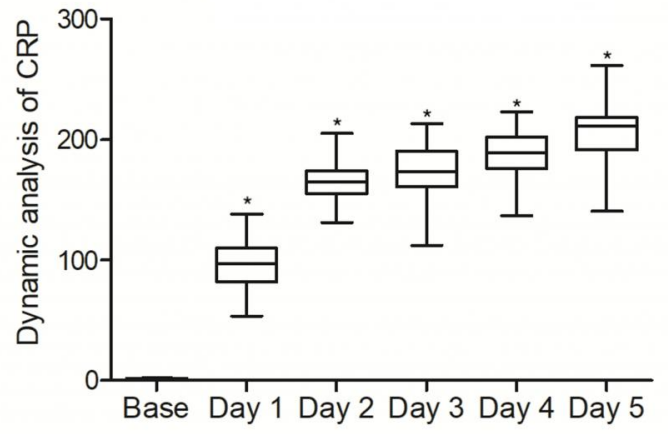

Noninfection, $\mathrm{N}=58$

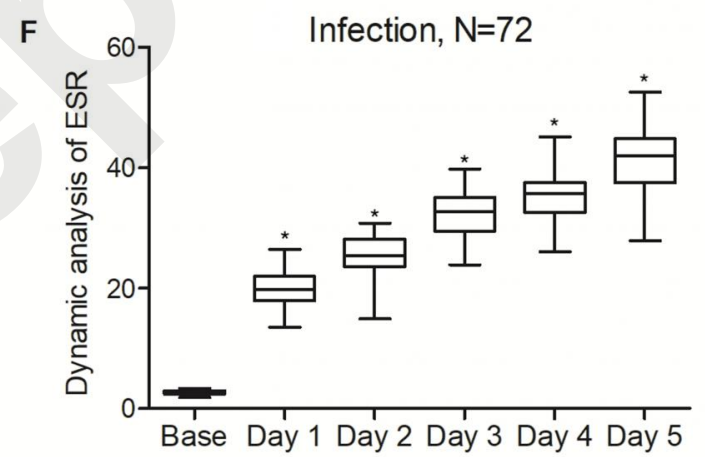

Figure 2

PCT might act as a predictive and diagnostic biomarker of post-operative infection

A: PCT expression in non-infection group showed no obvious difference from day 0 to day 5

B: PCT expression in infection group was evidently increased from day 1 to day 5 after surgery in a time-dependent manner

C: CRP expression in non-infection group was evidently increased from day 1 to day 5 after surgery in a time-dependent manner

D: CRP expression in infection group was evidently increased from day 1 to day 5 after surgery in a time-dependent manner

E: Level of ESR in non-infection group was significantly up-regulated from day 1 to day 5 after surgery in a time-dependent manner

F: Level of ESR in infection group was significantly up-regulated from day 1 to day 5 after 
surgery in a time-dependent manner 
A

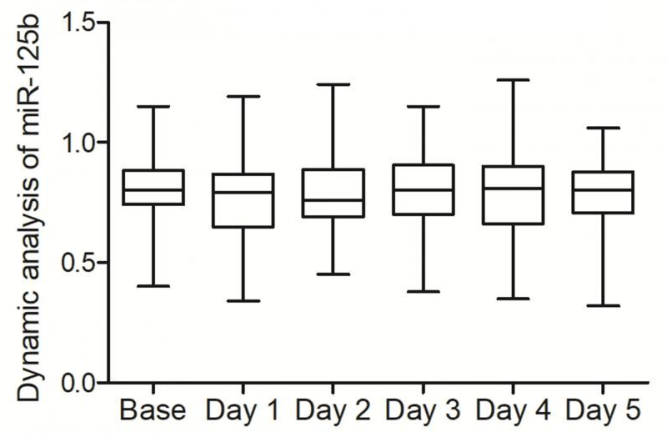

B

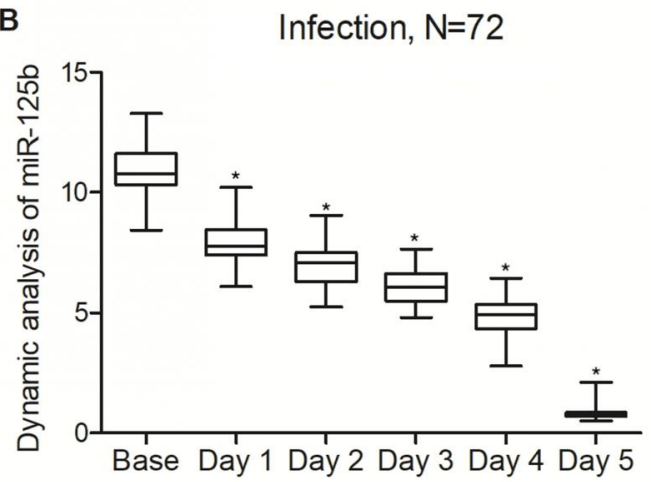

D

Infection, $\mathrm{N}=72$

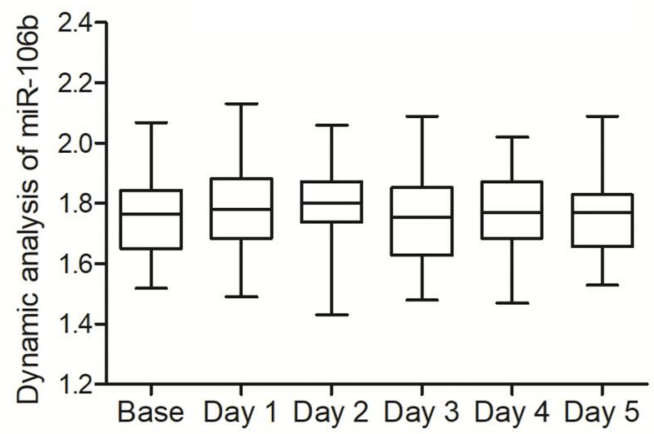

$E$

Noninfection, $\mathrm{N}=58$

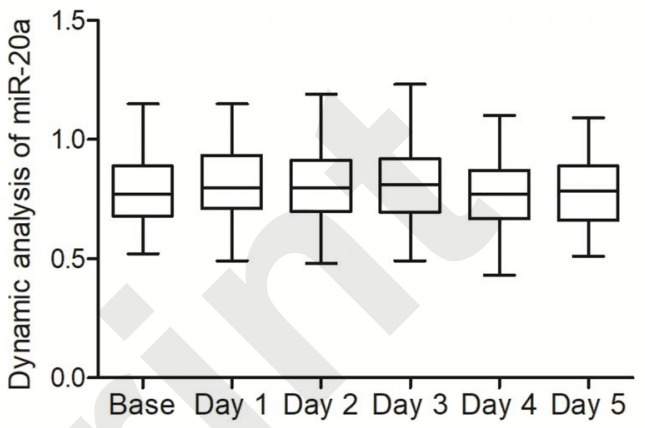

$\mathbf{F}$

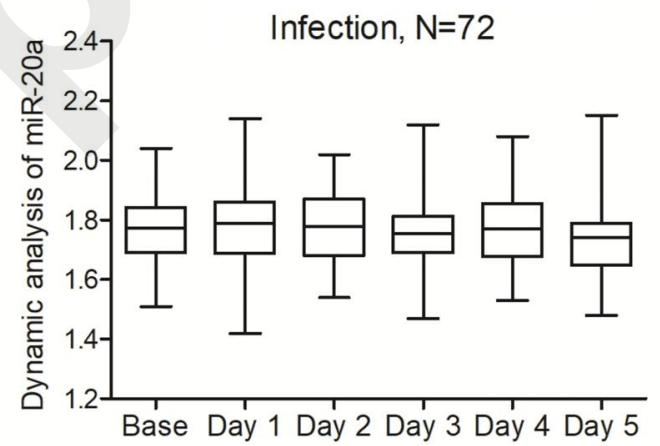

Figure 3

miR-125b was involved in post-operative infection

A: miR-125b level in non-infection group showed no obvious difference from day 0 to day 5

B: miR-125b level in infection group was evidently decreased from day 1 to day 5 after surgery in a time-dependent manner

C: miR-106b level in non-infection group showed no obvious difference from day 0 to day 5

$\mathrm{D}$ : miR-106b level in infection group showed no obvious difference from day 0 to day 5

$\mathrm{E}$ : Level of miR-20a in non-infection group remained stable from day 0 to day 5

$\mathrm{F}$ : Level of miR-20a in infection group remained stable from day 0 to day 5 

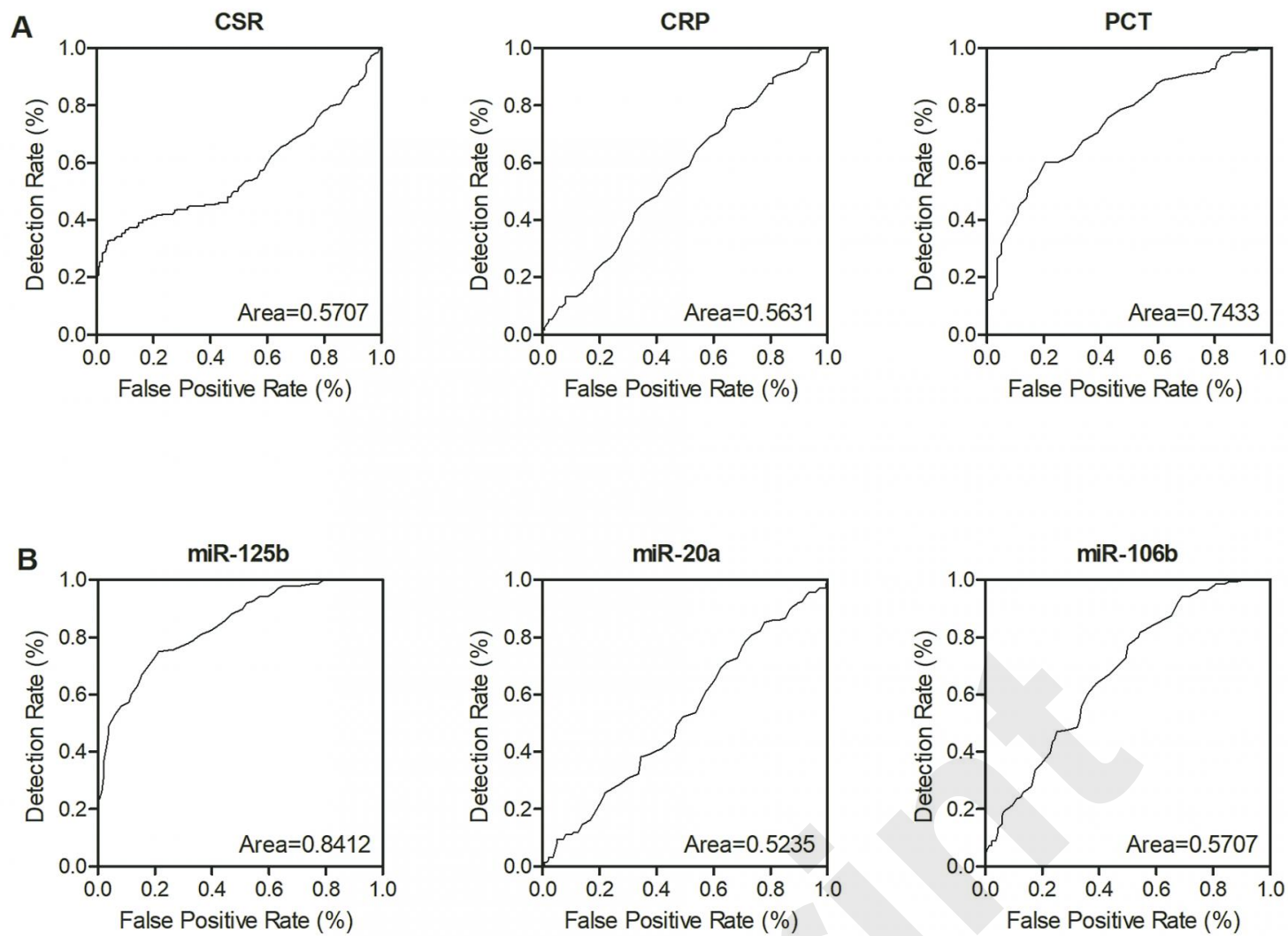

Figure 4

MiR-125b and PCT were associated with post-operative infection

A: The AUC values of PCT, CRP, and ESR were $0.75,0.55$ and 0.55 , respectively

$\mathrm{B}$ : The AUC values of miR-125b, miR-106b and miR-20a were $0.85,0.5$ and 0.7 , respectively 
A

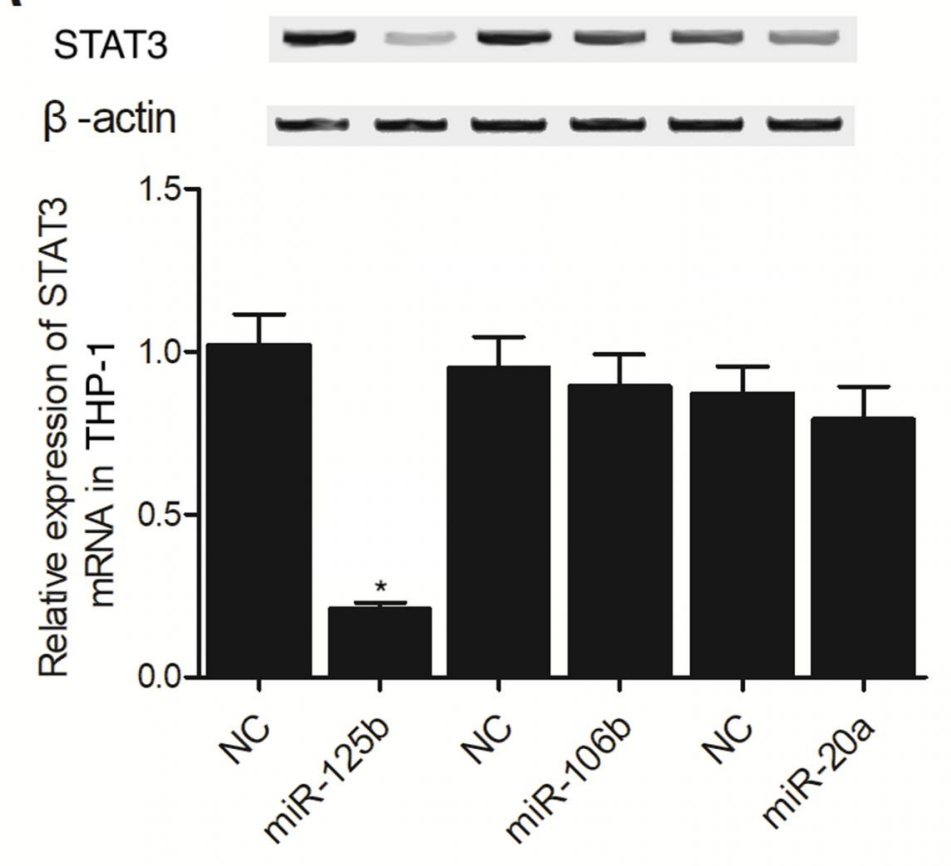

B

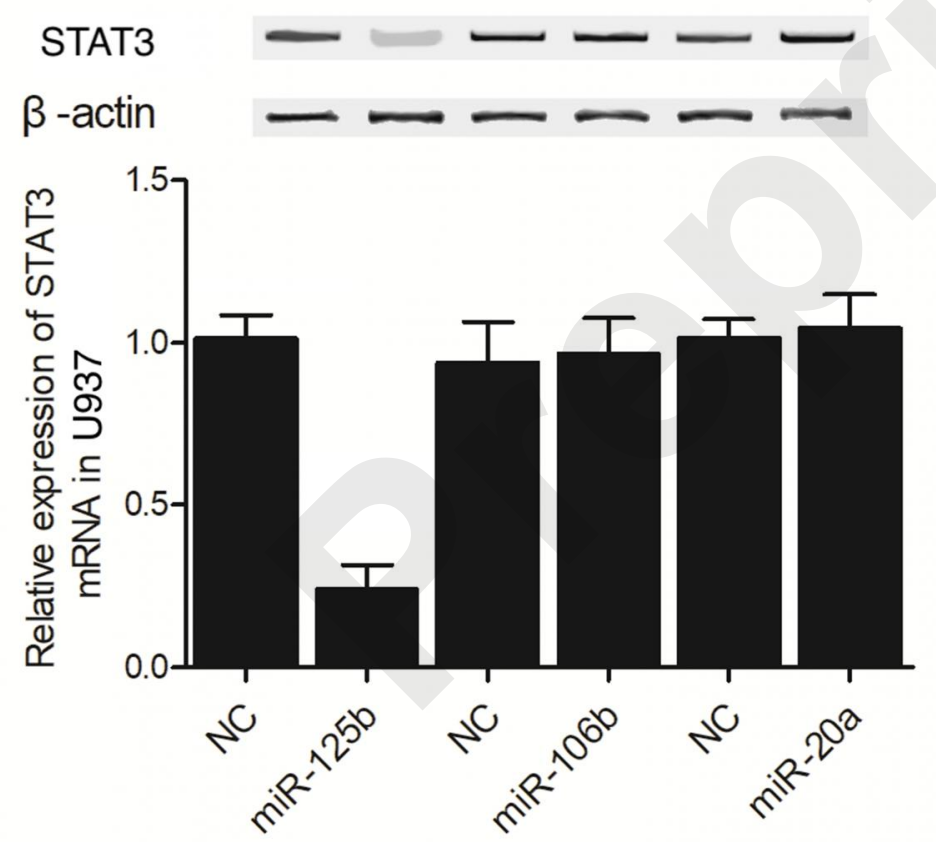

Figure 5

MiR-125b reduced STAT3 expression

A: The mRNA and protein levels of STAT3 in THP-1 cells was significantly reduced by the transfection with miR-125b mimics, while miR-106b and miR-20a mimics had no effect on STAT3 expression ( ${ }^{*}$ P value $<0.05$, vs. NC group; NC: negative control).

$B$ : The mRNA and protein levels of STAT3 in U937 cells was significantly reduced by the transfection with miR-125b mimics, while miR-106b and miR-20a mimics had no effect on STAT3 expression ( ${ }^{*} \mathrm{P}$ value $<0.05$, vs. NC group; NC: negative control). 
A

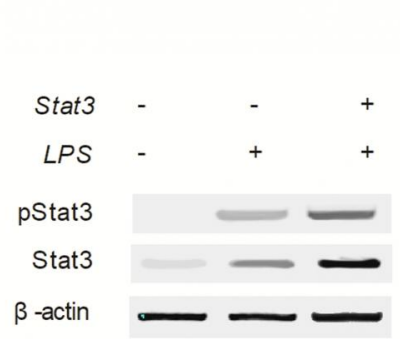

D

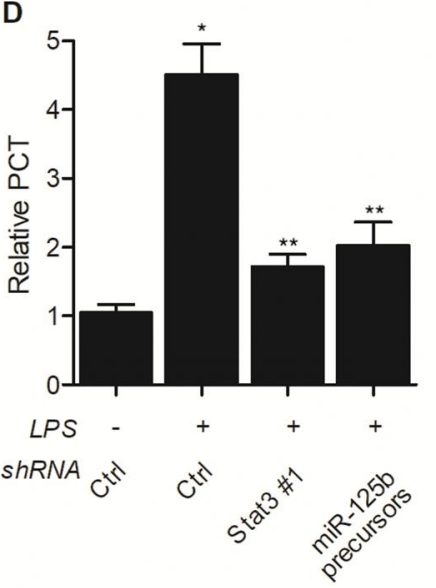

G

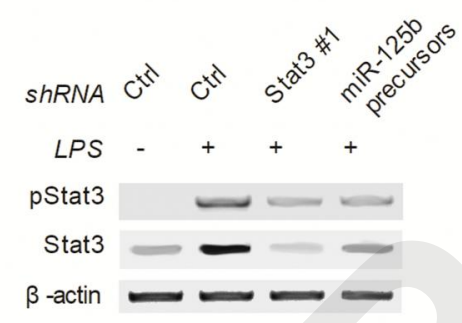

B

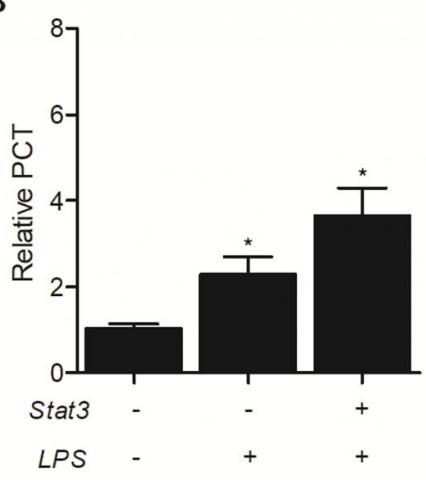

E

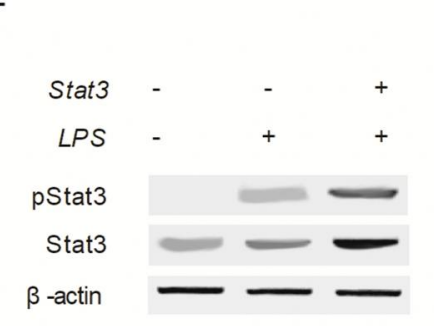

H

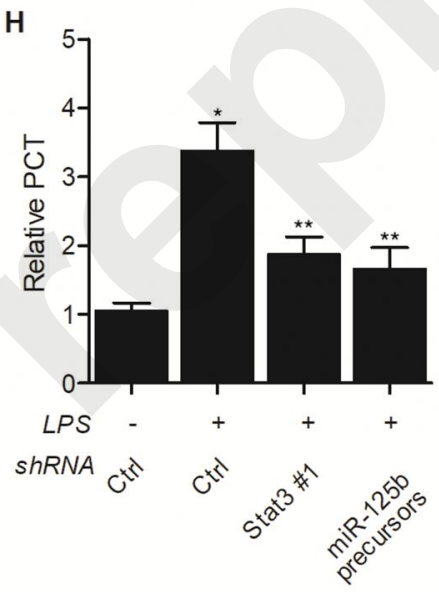

C

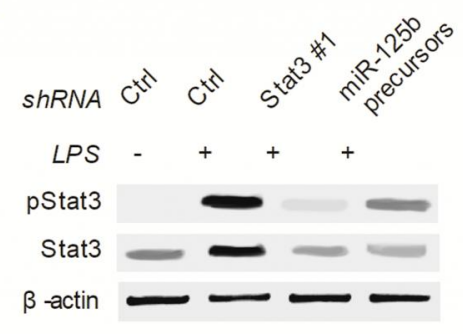

$\mathrm{F}$

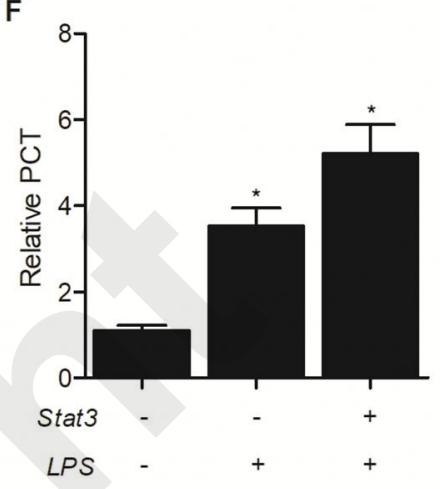

Figure 6

Activation of STAT3 increased PCT production

A: Levels of total STAT3 and phospho-STAT3 in THP-1 cells were increased by LPS treatment and further up-regulated by LPS + STAT3 treatment

B: PCT production in THP-1 cells was increased by LPS treatment and further up-regulated by LPS + STAT3 treatment ( ${ }^{*}$ P value $<0.05$, vs.Stat3 (-) LPS (-) group)

C: LPS-induced production of total STAT3 and phosphorylated STAT3 was repressed in THP-1 cells by shRNA-mediated STAT3 knockdown

D: ShRNA-mediated STAT3 knockdown repressed LPS-induced production of PCT in THP-1 cells ( ${ }^{*}$ value $<0.05$, vs.Stat3 (-) LPS (-) group; ${ }^{* *} P$ value $<0.05$, vs. LPS (+) Stat3 (-) group)

E: Levels of total STAT3 and phospho-STAT3 in U937 cells were increased by LPS 
treatment and further up-regulated by LPS + STAT3 treatment

F: PCT production in U937 cells was increased by LPS treatment and further up-regulated by LPS + STAT3 treatment ( ${ }^{*}$ value $<0.05$, vs.Stat3 (-) LPS (-) group)

G: LPS-induced production of total STAT3 and phosphorylated STAT3 was repressed in U937 cells by shRNA-mediated STAT3 knockdown

$\mathrm{H}$ : ShRNA-mediated STAT3 knockdown repressed LPS-induced production of PCT in U937 cells ( ${ }^{*}$ P value $<0.05$, vs.Stat3 (-) LPS (-) group; ${ }^{* *}$ P value $<0.05$, vs. LPS (+) Stat3 (-) group) 
A
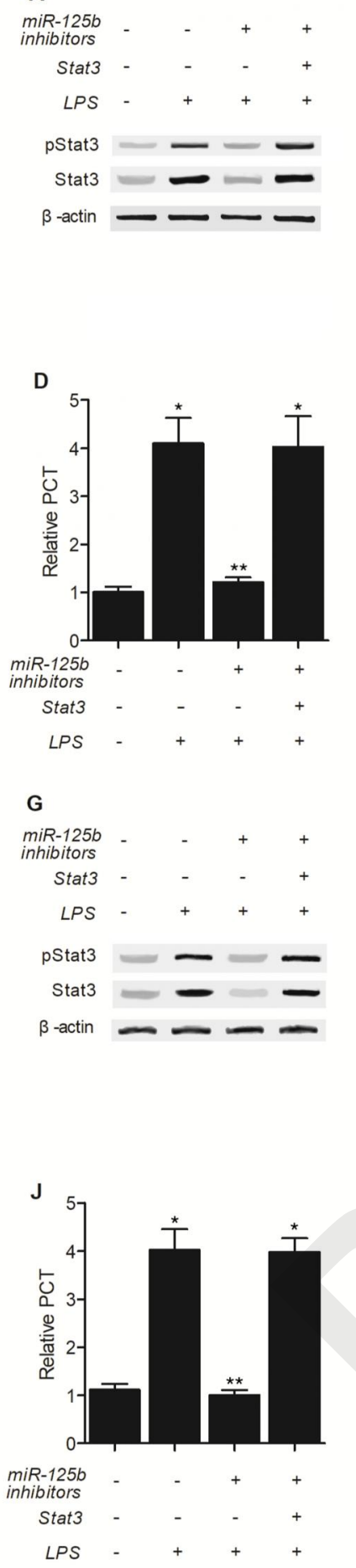

B

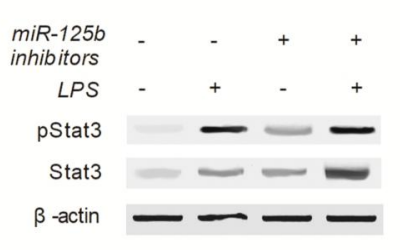

C

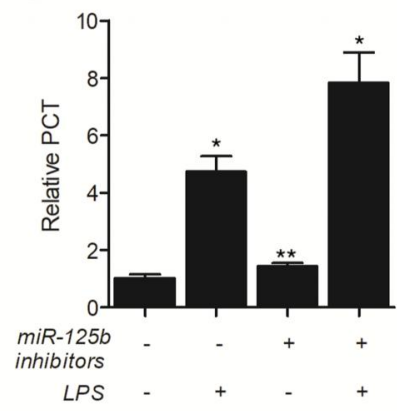

$E$
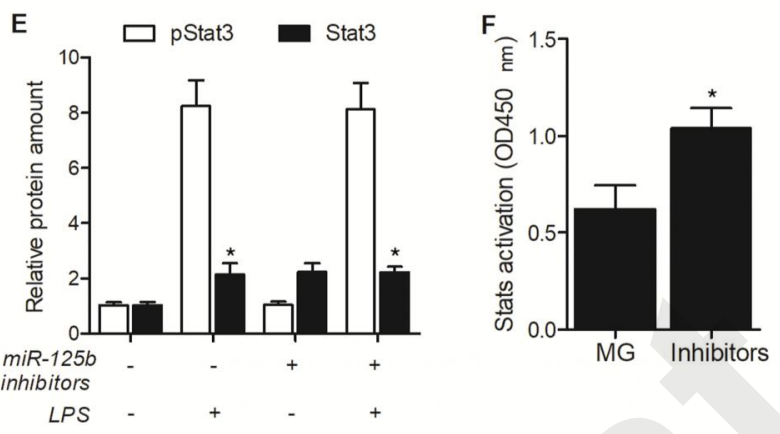

$\mathrm{H}$
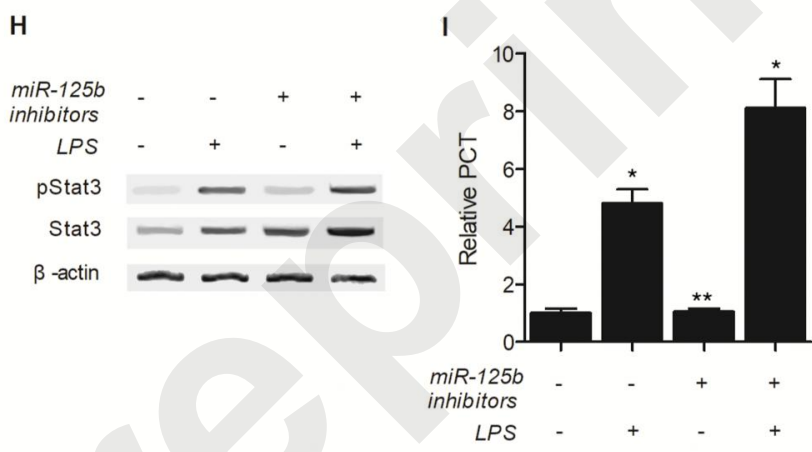

$\mathrm{K}$

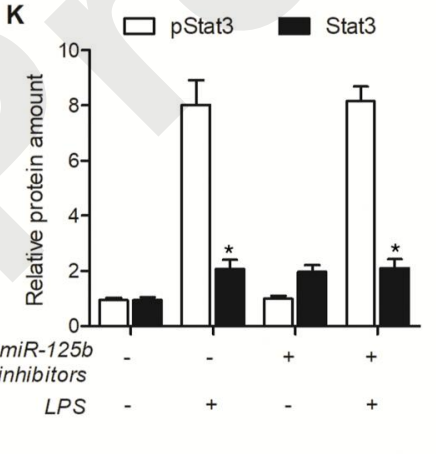

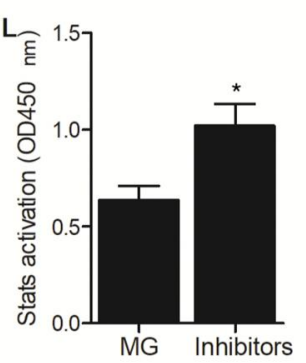

Figure 7

MiR-125b inhibited PCT expression by targeting STAT3

A: Levels of total STAT3 and phospho-STAT3 in THP-1 cells were decreased by miR-125b but increased by STAT3 treatment

B: LPS-induced production of phosphorylated STAT3 but not total STAT3 was repressed in THP-1 cells by miR-125b inhibitors but increased by STAT3 treatment C: PCT production in THP-1 cells was decreased by miR-125b but increased by STAT3 treatment ( ${ }^{*} \mathrm{P}$ value $<0.05$, vs. miR-125 inhibitors (-) LSP (-) group; ${ }^{* *} \mathrm{P}$ value $<0.05$, vs. miR-125 inhibitors(-) LSP (+) group) 
D: Inhibition of miR-125b increased the level of PCT in THP-1 cells ( ${ }^{*} P$ value $<0.05$, vs. miR-125 inhibitors (-) Stat3 (-) LSP (-) group; ${ }^{* *} \mathrm{P}$ value $<0.05$, vs. miR-125 inhibitors(-) Stat3 (-) LSP (+) group)

E: DNA-binding activity of STAT3 was obviously increased in THP-1 cells by miR-125b inhibitors

F: Levels of total STAT3 and phospho-STAT3 in THP-1 cells were decreased by miR-125b but increased by STAT3 treatment

G: PCT production in THP-1 cells was decreased by miR-125b but increased by STAT3 treatment

$\mathrm{H}$ : LPS-induced production of phosphorylated STAT3 but not total STAT3 was repressed in THP-1 cells by miR-125b inhibitors but increased by STAT3 treatment

I: Inhibition of miR-125b increased the level of PCT in THP- 1 cells $\left({ }^{*} P\right.$ value $<0.05$, vs. miR-125 inhibitors (-) LSP (-) group; ${ }^{* *} \mathrm{P}$ value $<0.05$, vs. miR-125 inhibitors(-) LSP (+) group)

J: DNA-binding activity of STAT3 was obviously increased in THP-1 cells by miR-125b inhibitors 